\title{
ORIGINAL ARTICLE INTERACTION OF TOBACCO ABUSE WITH OTHER CONVENTIONAL RISK FACTORS OF CARDIOVASCULAR RISK - SYNERGISTIC IMPACT OR AN INDEPENDENT MENACE?
}

\author{
Arslan Masood ${ }^{1}$, Noor Dastgir'1, Inam Ur Rehman'1, Junaid Rehman', Aleena Khan', \\ Rimsha Munir ${ }^{1}$, Syed Ali Abbas ${ }^{1}$ \\ ${ }^{1}$ Allama Iqbal Medical College / Jinnah Hospital, Lahore, Pakistan
}

\begin{abstract}
Objectives: To determine the prevalence, patterns and behavioural attributes of tobacco abuse in patients of acute coronary syndrome (ACS). Furthermore, to assess the interaction of tobacco abuse with other conventional risk factors of cardiovascular disease (CVD).

Methodology: This observational study included 230 consecutive patients with ACS. Data was collected regarding total duration and extent of tobacco consumption, "tobacco addiction" and various behavioural patterns related to it. Risk factors profile was acquired for hypertension, diabetes, obesity, family history of premature CVD and dyslipidemia. Odds ratios (OR) with 95\% confidence intervals (CI) for these risk factors were calculated for tobacco abusers compared to non-abusers.

Results: Among the study population, 63(27.4\%) were active tobacco users. Urban residents had lesser odds of being tobacco abusers compared to non-urban residents $(0.49,[0.27-0.89])$. Tobacco abusers had a lower prevalence of hypertension compared to non-abusers $(0.44$ [0.24 - 0.81]). A similar trend was observed for diabetes, obesity and dyslipidemia, however, the differences could not reach significance thresholds. Cigarette smoking was the commonest mode of tobacco consumption (90.5\%). "Tobacco addiction" could be attributed to $84.1 \%$ of abusers. Most $(82.5 \%)$ were willing to give up tobacco abuse and $63.3 \%$ had already made attempts at quitting.

Conclusion: About one-third of ACS patients were tobacco abusers with the majority being tobacco addicts. Tobacco abuse was observed to be independently implicated as a risk factor in ACS patients. Furthermore, tobacco abuse was inversely related to hypertension trans lating into a sub-multiplicative / additive impact of hypertension as a risk factor.
\end{abstract}

Keywords: Tobacco, Tobacco addiction, Cigarette, CVD risk factors, Acute coronary syndrome

Citation: Masood A, Dastgir N, Rehman IU, Rehman J, Khan A, Munir R, Abbas SA. Interaction of Tobacco Abuse with other Conventional Risk Factors of Cardiovascular Risk - Synergistic Impact or an Independent Menace?. Pak Heart J. 2021;54(04):352-356. DOI: https://doi.org/10.47144/phj.v54i4.2121

\section{INTRODUCTION}

Tobacco use is one of the major causes of death worldwide and the number of patients who die of smoking-related complications is expected to continue rising. The prevalence of tobacco use has not declined significantly in developing countries. Asia-Pacific region shared $65 \%$ of global cigarette sales by volume in 2018 making it the biggest cigarette consuming market worldwide. ${ }^{1}$ In addition to cigarettes, there are several unique modes of tobacco consumption in South-Asian countries, including pipe smoking, water-pipes and various chewable tobacco formulations. ${ }^{2}$ In Pakistan, twenty-three percent of men and five percent of women use any type of tobacco; cigarette smoking being the most common form (22\% of men and 3\% of women). ${ }^{3}$ A study conducted on non-atherosclerotic Pakistani patients showed higher lifetime risk of cardiovascular diseases (CVD) in males (49.4\%) compared to females
$(31.9 \%) .{ }^{4}$ Furthermore, a linear relationship has been observed between number of cigarettes smoked and risk of developing CVD in studies conducted in Pakistan. ${ }^{5}$

Hypertension, diabetes, obesity, sedentary life-style and dyslipidemias are other well-documented risk factors for CVD ${ }^{6-7}$ Several mechanisms are implicated in smoking as a cause of CVD including endothelial damage, increase platelet aggregation, inflammation, oxidative stress and serum lipid derangements. ${ }^{8}$ Other risk factors, particularly diabetes for instance, lead to accelerated atherosclerosis and subsequent development of CVD. ${ }^{9}$ Raised serums levels of total cholesterol and low-density lipoproteins are involved in the initiation of plaque formation and progression whereas smoking is linked to modification of these plaques morphologies and changes in the plaque stability. ${ }^{10}$ 
In addition to being a major CVD risk factor, tobacco abuse also impacts and alters other risk factors by various mechanisms, thus making the analysis and understanding of the joint-affect of these risk factors complicated. The potential synergistic or additive effect of tobacco abuse on other conventional risk factors remains variable in the literature. Tobacco abuse may be considered a component of a generalized unhealthy behavioral pattern associated with obesity, diabetes, metabolic syndrome, sedentary lifestyle, hypertension and dyslipidemia - all imposing additive cumulative impacts on the development of CVD. On the other hand, it may independently lead to raised CVD risk in the absence of other major risk factors. The interplay of tobacco abuse and other concomitant risk factors remains unclear particularly in South Asians considering less robust tobacco control programs and ever-rising trends of other concomitant CVD risk factors. The current study was carried out to determine the prevalence, patterns and behavioral attributes of tobacco abuse in patients of ACS. Additional objective was to assess the interaction of tobacco abuse with other conventional risk factors of CVD.

\section{METHODOLOGY}

This Cross sectional study included 230 consecutive patients of ACS - unstable angina (UA), non-ST elevation myocardial infarction (NSTEMI) and STelevation myocardial infarction (STEMI) over 6 months (August 2020 to February 2021) at Cardiology Department Jinnah hospital, Lahore. A sample size of 273 was calculated considering a $5 \%$ margin of error, $95 \%$ confidence level and a $23 \%$ expected prevalence of tobacco abuse in the local population. A final sample of 230 was considered and analyzed after exclusion of cases with insufficient data. Formal approval was sought from the institutional ethical review board and a validated proforma was used to gather data from the patients who consented for the study. Data was gathered regarding the demographic profiles, tobacco abuse patterns and other CVD risk factor profiles.

A criterion of current tobacco consumption or its use over most of the days over the last six-months was used to label "tobacco abuse". Data was collected regarding the total duration of tobacco consumption and the extent of daily use. "Tobacco addiction" was labeled if the patient agreed to have at least one failed attempt at quitting the abuse, had progressively increasing consumption or reported having withdrawal symptoms. Data was stratified according to gender, age, ACS types, educational status, marital status, geographic and domestic distributions and the prevalence of tobacco abuse was compared among these strata. Further behavioral patterns were assessed including the type of tobacco consumption, reasons to start tobacco, intention to quit and the number of attempts made at quitting the abuse.

Other conventional risk factors profile was acquired for hypertension (resting blood pressure of $\geq 140$ $\mathrm{mmHg}$ systolic or $\geq 90 \mathrm{mmHg}$ diastolic), diabetes (glycated hemoglobin $\geq 6.5 \%$ ), obesity (basal metabolic index $\geq 30 \mathrm{Kg} / \mathrm{m} 2$ ), family history of premature coronary artery disease (in first degree relatives at age $<45$ years for males and 55 years for females) and dyslipidemia (total serum cholesterol $\geq$ $200 \mathrm{mg} / \mathrm{dl}, \mathrm{LDL} \geq 130 \mathrm{mg} / \mathrm{dl}$, triglycerides $>200$ $\mathrm{mg} / \mathrm{dl}$ or $\mathrm{HDL}<35 \mathrm{mg} / \mathrm{dl}$ analyzed from serum samples after 10 to 12 hours of fasting). The data was analyzed by the software Statistical Package for Social Sciences (SPSS) version 23. The prevalence of these conventional risk factors was compared among tobacco abusers and non-abusers by Pearson's chisquare test considering a p-value of $\leq 0.05$ as statistically significant. ORs with $95 \%$ CIs for these risk factors were calculated for tobacco abusers compared to non-abusers.

\section{RESULTS}

The mean age of the study population was $54.6 \pm$ 11.1 years (69.6\% males and $30.4 \%$ females). STEMI was the most frequent admission diagnosis $(50.9 \%)$, followed by unstable angina $(33 \%)$ and non-ST elevation MI (16.1\%). Among the study population, $63(27.4 \%)$ were active tobacco users. Tobacco abuse did not show significant among ACS types $(\mathrm{p}=0.31)$. Most of the participants $(65.6 \%)$ were 40 to 60 years old and tobacco abuse did not vary statistically among the age strata $(\mathrm{p}=0.24)$. There was an equal representation of educational strata and tobacco abuse did not differ among them $(p=0.80)$. The study participants belonged to the major city $(65.2 \%)$, its adjoining towns and villages $(11.3 \%)$ as well as other cities of the province $(23.5 \%)$. Residents of urban areas had lesser odds of being tobacco abusers compared to non-urban residents $(\mathrm{OR}=0.49,95 \%$ CIs $0.27-0.89, \mathrm{p}=$ 0.014) (Table 1).

Among other CVD risk factors, hypertension was the most prevalent $(52.8 \%)$, followed by diabetes mellitus $(37.0 \%)$, obesity $(35.7 \%)$, family history of premature CVD (24.5\%) and dyslipidemia (24.2\%) (Figure 1). Tobacco abusers had a lower prevalence of hypertension compared to non-abusers $(\mathrm{OR}=$ $0.44,95 \%$ CIs $0.24-0.81, \mathrm{p}=0.006)$. A similar trend was observed regarding diabetes, obesity and dyslipidemia where tobacco abusers had lesser prevalence of these CVD risk factors compared to 
non-abusers, however, the differences could not reach the significance thresholds (diabetes in tobacco abusers OR $=0.5795 \%$ CIs $0.30-1.08 \mathrm{p}=$ 0.056 ; obesity OR $=0.5495 \%$ CIs $0.19-1.58, \mathrm{p}=$ 0.192 ; dyslipidemia OR $=0.2795 \%$ CIs $0.06-1.35$ $\mathrm{p}=0.085)$. Prevalence of family history of premature CVD was similar between tobacco abusers and non-abusers $(\mathrm{OR}=1.395 \%$ CIs $0.65-$ $2.61 \mathrm{p}=0.285)($ Figure 1$)$

Table 1: Demographic profiles of study participants with comparisons between tobacco abusers and nonusers

\begin{tabular}{|c|c|c|c|c|}
\hline & Total & Tobacco Users & Non-Tobacco Users & P-values \\
\hline \multicolumn{5}{|l|}{ Gender } \\
\hline Males & $160(69.6 \%)$ & $60(95.2 \%)$ & $100(60 \%)$ & \multirow{2}{*}{$<0.001$} \\
\hline Females & $70(30.4 \%)$ & $03(4.8 \%)$ & $67(40 \%)$ & \\
\hline \multicolumn{5}{|l|}{ Admission diagnosis } \\
\hline Unstable angina & $76(33.0 \%)$ & $16(25.4 \%)$ & $60(35.9 \%)$ & \multirow{3}{*}{0.31} \\
\hline Non-ST elevation MI & $37(16.1 \%)$ & $11(17.5 \%)$ & $26(15.6 \%)$ & \\
\hline ST elevation MI & $117(50.9 \%)$ & $36(57.1 \%)$ & $81(48.5 \%)$ & \\
\hline \multicolumn{5}{|l|}{ Age strata } \\
\hline 40 years or younger & $24(10.4 \%)$ & $9(14.3 \%)$ & $15(9 \%)$ & \multirow{3}{*}{0.24} \\
\hline Between 40 to 60 years & $151(65.6 \%)$ & $43(68.2 \%)$ & $108(64.7 \%)$ & \\
\hline 60 years and older & $55(24.0 \%)$ & $11(17.5 \%)$ & $44(26.3 \%)$ & \\
\hline \multicolumn{5}{|l|}{ Education } \\
\hline Uneducated & $66(28.7 \%)$ & $17(27 \%)$ & $49(29.3 \%)$ & \multirow{4}{*}{0.80} \\
\hline Primary & $54(23.5 \%)$ & $17(27 \%)$ & $37(22.2 \%)$ & \\
\hline High school & $59(25.6 \%)$ & $17(27 \%)$ & $42(25.1 \%)$ & \\
\hline Bachelors or higher & $51(22.2 \%)$ & $12(19 \%)$ & $39(23.4 \%)$ & \\
\hline \multicolumn{5}{|l|}{ Marital status } \\
\hline Married & $228(99.1 \%)$ & $63(100 \%)$ & $165(98.8 \%)$ & \multirow{2}{*}{$>0.999$} \\
\hline Unmarried & $2(0.9 \%)$ & $0(0 \%)$ & $2(1.2 \%)$ & \\
\hline \multicolumn{5}{|l|}{ Geographic distribution } \\
\hline Main city & $150(65.2 \%)$ & $33(52.4 \%)$ & $117(70.1 \%)$ & \multirow{3}{*}{0.042} \\
\hline Adjacent towns and villages & $26(11.3 \%)$ & $10(15.9 \%)$ & $16(9.6 \%)$ & \\
\hline Other cities & $54(23.5 \%)$ & $20(31.7 \%)$ & $34(20.4 \%)$ & \\
\hline \multicolumn{5}{|l|}{ Domestic distribution } \\
\hline Urban residents & $109(47.4 \%)$ & $22(34.9 \%)$ & $87(52.1 \%)$ & \multirow{2}{*}{0.014} \\
\hline Non-urban residents & $121(52.6 \%)$ & $41(65.1 \%)$ & $80(47.9 \%)$ & \\
\hline
\end{tabular}

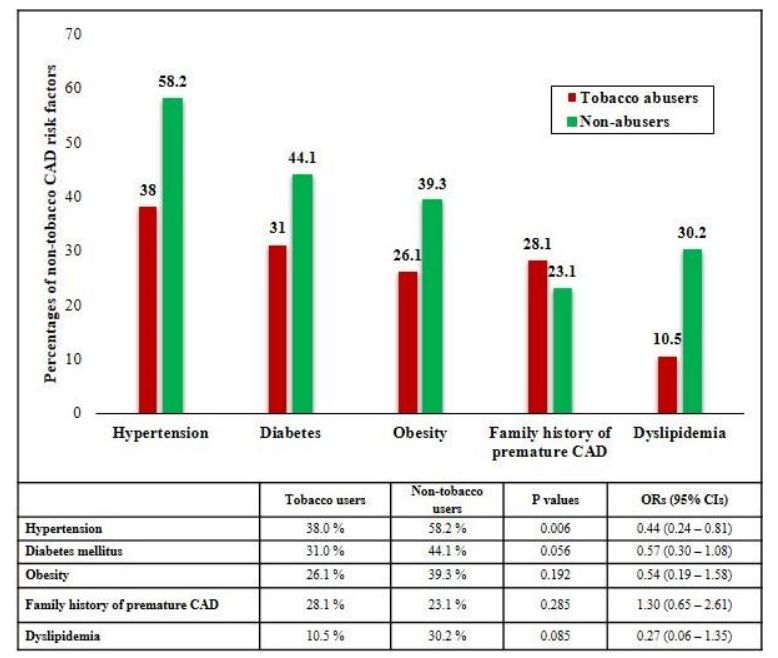

Figure 1: Comparison of non-tobacco CAD risk factors prevalence among tobacco abusers and non-tobacco abusers

Cigarette smoking was the commonest mode of tobacco consumption $(90.5 \%)$, followed by pipesmoking (3.2\%) and tobacco chewing (6.3\%). Among tobacco abusers, the mean duration of consumption was $24.2 \pm 11.5$ years with a mean daily consumption of $18.5 \pm 9.5$ cigarettes. "Tobacco addiction" could be attributed to $84.1 \%$ of abusers as per specified criteria.

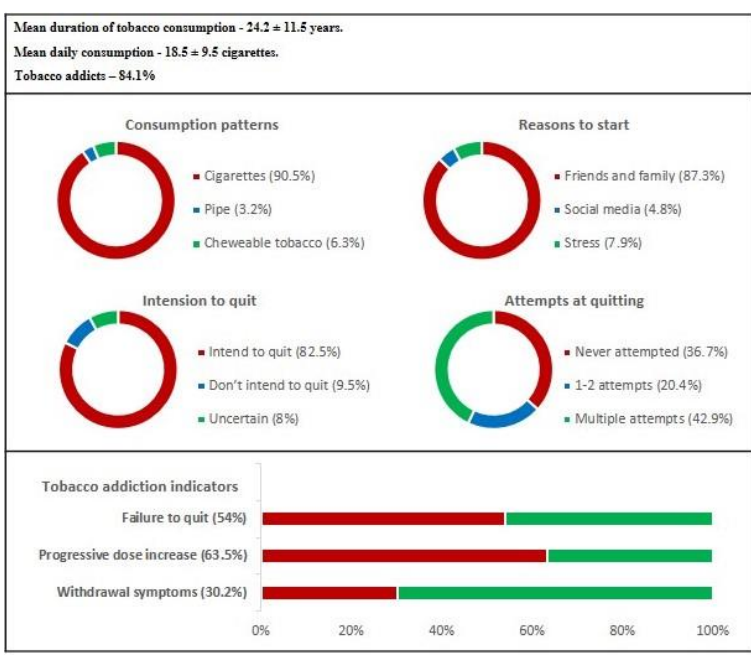

Figure 2: Consumption patterns, social, behavioural and addiction attributes of tobacco abuse 
Most abusers $(87.3 \%)$ could link their initiation of tobacco consumption to be influenced by their friends or family members. When asked about their intentions and plans to quit tobacco abuse, the majority (82.5\%) agreed to having their intentions to quit (Figure 2).

\section{DISCUSSION}

The study was based on data gathered from cases of evident ischemic heart disease in the form of ACS with a large fraction of patients suffering from STEMI $(50.9 \%)$. The idea was to assess various patterns, behaviours and interactions of tobacco abuse with non-tobacco CVD risk factors in this extreme exhibit subset of the CVD spectrum. The study population had representation from a broad age range and an almost equal distribution of various educational levels. The prevalence of tobacco abuse did not show a significant difference among the age strata or educational groups ( $\mathrm{p}=0.24$ and 0.80 respectively).

In a recent in-depth analysis of complex interactions between smoking and other CVD risk factors by Lubin et al., tobacco smoking showed synergistic/multiplicative impact on the relative risk (RR) of CVD with hypertension, total cholesterol, low-density lipoproteins; a non-synergistic / additive risk with obesity, diabetes, and high-density lipoproteins. ${ }^{11}$ Another interesting finding of the study was observing a low-quantity and prolonged smoking to be more deleterious than heavier smoking for shorter durations. ${ }^{11}$ In various observational studies and the EPOCH-JAPAN study a multiplicative interaction has been observed between smoking and hypertension. ${ }^{12,13}$ Smokers were found more likely to be hypertensive in previous Pakistani data. ${ }^{14}$ In our study, tobacco non-users were more likely to be hypertensives compared to abusers suggesting a submultiplicative interaction between tobacco abuse and hypertension in patients of ACS. Tobacco abuse has been inversely related to hypertension in literature as well. ${ }^{15}$ Tobacco abusers who suffer from ACS, tend to be younger and show lower burden of other factors of CV morbidity and mortality. ${ }^{16}$

Regarding diabetes-related risk of CVD, non-smokers had a greater risk compared to smokers as per findings of a meta-analysis of 102 European studies thus showing a sub-multiplicative / supra-additive association. ${ }^{17}$ However, the diabetes-related risk of CVD was similar according to smoking status according to a Japanese study. ${ }^{18}$ Our study suggested a sub-multiplicative interaction between diabetes and tobacco abuse, although the significance level was not reached. Variable interactions have been reported in the literature for metabolic factors and smoking. A multiplicative association has been suggested due to similar RR for BMI by smoking status or quantity of daily cigarette consumptions, ${ }^{18}$ while a submultiplicative interaction was suggested due to higher RRs for BMI in non-smokers. ${ }^{19}$ A supra-multiplicative association was found on the other hand in an AsiaPacific region's meta-analysis where RR of CVD per unit BMI was greater in current smokers compared to non-smokers. ${ }^{20}$ In our study, the prevalence of obesity was not statistically different among the tobacco abuse groups.

Most of the data regarding interaction of dyslipidaemias and smoking from meta-analysis, pooled analysis from EPOCH-JAPAN, European and North American cohorts is in favour of a multiplicative association where smoking status did not affect the RRs by TC. ${ }^{12,21,22}$ However, a metaanalysis based on 34 studies from Asia-Pacific region found a greater risk in smokers per unit increase in TC compared to non-smokers. ${ }^{23}$ In our study, the prevalence of dyslipidaemia was not statistically different among tobacco abuse groups.

Several important behavioral attributes were drawn from this study. A large proportion of tobacco abusers $(84.1 \%)$ were found "addicts" to this menace. Most tobacco abusers $(87.3 \%)$ attributed the influence of their family and friends as the single most frequent contributory factor to start using tobacco. However, the positive side of the picture was the encouraging percentage of tobacco abusers who were willing to give up this habit $(82.5 \%)$ and a little less than twothirds $(63.3 \%)$ had already made attempts at quitting.

The organizational limitations of the study included its lack of a control group, non-randomized nature and being single-centred. Tobacco abuse patterns may vary according to diverse ethnicities and large-scale multi-centre or multi-ethnic population-based studies are suggested to cater for these variations in future research.

\section{CONCLUSION}

About one-third of ACS patients were tobacco abusers with the majority being tobacco addicts. Tobacco abuse was observed to be independently implicated as a risk factor in ACS patients. Furthermore, tobacco abuse was inversely related to hypertension translating into a submultiplicative / additive impact of hypertension as a risk factor.

\section{AUTHORS' CONTRIBUTION}

AM: Concept and design, data acquisition, interpretation, drafting, final approval, and agree to be accountable for all aspects of the work. ND, IUR, JR, AK, RM, SAA: Data acquisition, interpretation, drafting, final approval and agree to be accountable for all aspects of the work. 
Conflict of interest: Authors declared no conflict of interest.

\section{REFERENCES}

1. Cigarettes in Asia Pacific. Euromonitor, Dec. 2020. Available at: www.euromonitor.com/cigarettes-in-asia-pacific/report

2. Masud H, Oyebode O. Inequalities in smoking prevalence: a missed opportunity for tobacco control in Pakistan. J Public Health (Oxf). 2018;40(2):271-8.

3. National Institute of Population Studies (NIPS) [Pakistan] and ICF. 2019. Pakistan demographic and health survey 2017-18 Islamabad, Pakistan, and Rockville, Maryland, USA: NIPS and ICF.

4. Ashraf T, Achakzai AS, Farooq F, Memon MA, Mengal N, Abbas $\mathrm{KY}$, et al. Estimating risk of atherosclerotic cardiovascular diseases in non-atherosclerotic Pakistani patients: Study conducted at National Institute of Cardiovascular Diseases, Karachi, Pakistan. J Pak Med Assoc. 2017;67(4):494-8.

5. Barolia R, Sayani A H. Risk factors of cardiovascular disease and its recommendations in Pakistani context. J Pak Med Assoc. 2017; 67(11):1723-9.

6. Chan YY, Sahril N, Rezali MS, Kuang Kuay L, Baharudin A, Abd Razak MA, et al. Self-reported modifiable risk factors of cardiovascular disease among older adults in Malaysia: A Crosssectional study of prevalence and clustering. Int J Environ Res Public Health. 2021;18(15):7941.

7. Bays HE, Taub PR, Epstein E, Michos ED, Ferraro RA, Bailey $\mathrm{AL}$, et al. Ten things to know about ten cardiovascular disease risk factors. Am J Prev Cardiol. 2021;5:100-149.

8. Kotani K, Miura K. Smoking and oxidised high-density lipoprotein: a preliminary report. Arch Med Sci Atheroscler Dis. 2021;6:e28-e29.

9. Strain WD, Paldánius PM. Diabetes, cardiovascular disease and the microcirculation. Cardiovasc Diabetol. 2018;17(1):57.

10. Gać P, Jaźwiec P, Mazur G, Poręba R. Exposure to cigarette smoke and the morphology of atherosclerotic plaques in the extracranial arteries assessed by computed tomography angiography in patients with essential hypertension. Cardiovasc Toxicol. 2017;17(1):67-78.

11. Lubin JH, Couper D, Lutsey PL, Yatsuya H. Synergistic and nonsynergistic associations for cigarette smoking and non-tobacco risk factors for cardiovascular disease incidence in the atherosclerosis risk in communities (ARIC) study. Nicotine Tob Res. 2017;19(7):826-35.

12. Nakamura $\mathrm{K}$, Nakagawa $\mathrm{H}$, Sakurai M, Murakami $\mathrm{Y}$, Irie F, Fujiyoshi A, et al; EPOCH-JAPAN Research Group. Influence of smoking combined with another risk factor on the risk of mortality from coronary heart disease and stroke: pooled analysis of 10 Japanese cohort studies. Cerebrovasc Dis. 2012;33(5):480-91.

13. Ge Z, Hao Y, Cao J, Li J, Chen J, Huang J, Wu X, Gu D. Does cigarette smoking exacerbate the effect of blood pressure on the risk of cardiovascular and all-cause mortality among hypertensive patients? J Hypertens. 2012;30(12):2307-13.

14. Liaquat A, Javed Q. Current trends of cardiovascular risk determinants in Pakistan. Cureus. 2018;10(10):e3409.

15. Wang M, Li W, Zhou R, Wang S, Zheng H, Jiang J, et al. The paradox association between smoking and blood pressure among half million Chinese people. Int J Environ Res Public Health. 2020;17(8):2824

16. Coutinho Cruz M, Illão Moreira R, Abreu A, Timóteo AT, Sá Carvalho R, Ferreira L, et al. The smoker's paradox in acute coronary syndrome: Is it real? Rev Port Cardiol (Engl Ed). 2018;37(10):847-55

17. Emerging Risk Factors Collaboration, Sarwar N, Gao P, Seshasai SR, Gobin R, Kaptoge S, Di Angelantonio E, et al. Diabetes mellitus, fasting blood glucose concentration, and risk of vascular disease: a collaborative meta-analysis of 102 prospective studies. Lancet. 2010;375(9733):2215-22.

18. Hata J, Doi Y, Ninomiya T, Fukuhara M, Ikeda F, Mukai N, et al. Combined effects of smoking and hypercholesterolemia on the risk of stroke and coronary heart disease in Japanese: the Hisayama study. Cerebrovasc Dis. 2011;31(5):477-84

19. Tobias D, Pan A, Hu FB. BMI and mortality among adults with incident type 2 diabetes. N Engl J Med. 2014;370(14):1363-4.

20. Asia Pacific Cohort Studies Collaboration. Impact of cigarette smoking on the relationship between body mass index and coronary heart disease: a pooled analysis of 3264 stroke and 2706 CHD events in 378579 individuals in the Asia Pacific region. BMC Public Health. 2009;9:294.

21. Huxley R, Nakamura K, Woodward M. Modification of the effect of lipids on the risk of cardiovascular diseases by cigarette smoking. J Clin Lipidology. 2010;5(3):413-20.

22. Lewington S, Whitlock G, Clarke R, Sherliker P, Emberson J, Halsey J, et al. Blood cholesterol and vascular mortality by age, sex, and blood pressure: a meta-analysis of individual data from 61 prospective studies with 55,000 vascular deaths. Lancet. 2007;370(9602):1829-39.

23. Nakamura K, Barzi F, Huxley R, Lam TH, Suh I, Woo J, et al. Asia Pacific Cohort Studies Collaboration. Does cigarette smoking exacerbate the effect of total cholesterol and high-density lipoprotein cholesterol on the risk of cardiovascular diseases? Heart. 2009;95(11):909-16.

\section{Address for Correspondence:}

Dr. Arslan Masood, Associate Professor, Department of Cardiology, Allama Iqbal Medical College / Jinnah Hospital, Lahore, Pakistan.

Email: dr_arslanmasood@hotmail.com 